\title{
Guía de la revista Creación \& Crítica (1971-1977)
}

\author{
Miguel Angel Rodríguez Rea \\ Departamento Académico de Literatura
}

In memoriam

Armando Rojas

Dirigida por Javier Sologuren, Ricardo Silva-Santisteban y Armando Rojas, Creación \& Crítica fue una de las revistas más importantes en su género en la década del ' 70 . En sus páginas se dieron cita autores de todas las lenguas y épocas, ya que estimuló de manera notable la traducción de textos antiguos y modernos. El aire cosmopolita de esta publicación recuerda otros esfuerzos como $3^{1}$ o Las Moradas $^{2}$ Debemos destacar en ese sentido la pulcritud de sus ediciones como también ta-amplia cobertura a las letras peruanas.

La presencia de un autor consagrado como Javier Sologuren (y sus colaboradores Ricardo Silva-Santisteban y Armando Rojas, poetas y traductores como él) será la clave para identificar a Creación \& Crítica en su propósito de entregar téxios y autores decantados, sin prejuicios artísticos de ninguna clase. Como lo ilustran los homenajes ofrecidos por la revista a Xavier Abril y a Emilio Adolfo Westphalen que, además de ser los primeros que se les tributaron entre nosotros, reflejan el reconocimiento generacional a dos figuras preclaras de la poesía peruana contemporánea.

1. Cf. Miguel Angel Rodríguez Rea, “Guía de la revista 3 (1939-1941)", Letras, № 95-96, 1998: 237-268.

2. Cf. Isabel Mármol Cornejo, Guía hemerográfica de Las Moradas. Miraflores: Universidad Nacional Mayor de San Marcos, Instituto Raúl Porras Barrenechea, 1974. ix, 39 h.

Letras (Lima), 97-98: 259-292, 1999 


\section{№ 1. ENERO DE 1971}

1. EIELSON, Jorge Eduardo

Poemas

Pp. [1-4].

C.: "Aura suprema...".- "Maldita noche....".-"Cuanto puede el aire..."."Tú".- "Metamorfosis (ejercicio poético)".- "Cero".- "Canon (poema giratorio con pájaros siglos esplendores etc.)".

2. MOTOKIYO, Seami

Izutsu.

Pp. $[5-10]$.

“... pieza de (teatro clásico japonés (noh)”.

Traducción por Javier Sologuren de la versión francesa del general Redondeau.

3. APOLLINAIRE, Guillaume

Onirocrítica.

Pp. [11-12].

aume y a."

"Texto originalmente piblieadeled E9I8eluegosformó parte del libro II

Traducción de Ricardo Silva-Santisteban.

4. EGUREN, José María

La sala ambarina.

Pp. [13-14].

Prosa poética.

Texto publicado anteriormente "en una limitadísima edición no venal de Ediciones de la Rama Florida en diciembre de 1969".

5. ROJAS, Armando

Sextinas y otros poemas de Carlos Germán Belli.

P. [15]. 
Reseña al libro del mismo título [Santiago de Chile: Editorial Universitaria, 1970].

\section{№ 2. FEBRERO DE 1971}

6. JOYCE, James

La última página de Finnegans Wake.

Pp. [1-2].

"Texto tomado de la primera reimpresión de la tercera edición de Finnegans Wake. London, Faber \& Faber Ltd., 1968: 626-628".

Traducción de Ricardo Silva-Santisteban.

7. SILVA-SANTISTEBAN, Ricardo

[Nota a la Traducción de "La última página de Finnegans Wake".]

Pp. [2-4].

8. SEFERIS, Georges

Delfos.

P. [4].

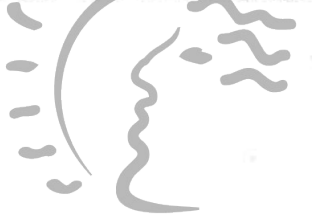

Poesía.

\section{Biblioteca de Letras}

Traducción de Javier Sologuren.

\section{"Jorge Puccinelli Converso"}

9. GUEVARA, Pablo

Poemas.

P. $[5-7]$.

C.: "El sol amotinado".- "Los desnudos".- "Llegado a este punto o canción caníbal de lo fugaz”.

10. LOAYZA, Luis

Dos versiones de una venganza.

Pp. [8-10].

Versiones en las Tradiciones peruanas de Ricardo Palma y en Historia general del Perú del Inca Garcilaso de la Vega. 
Ensayo.

11. OVIEDO, José Miguel

Cantos y poemas africanos modernos.

Pp. [11-13].

"Los cantos africanos fueron recogidos por León D. Damas. Leopold Sedar Senghor y David Diop son destacados poetas africanos contemporáneos".

C.: [Cantos:] "Cornudo satisfecho".- "El rencoroso".- "Las penas de Kodio".- [Poemas:] "Gozamos, mi amigo", Leopold Sedar Senghor.- "Africa", David Diop.

Traducción de J.M.O.

12. ROJAS, Armando

Vuelta a la otra margen.

Pp. [14-15].

Reseña a la antología del mismo título preparada por Mirko Lauer y Abelardo Oquendo [Lima: Casa de la Cultura del Perú, 1970].

\section{Biblioteca de Letras "Jorge Puccinelli fonirerso"}

13. THOMAS, Dylan

[Poemas.]

Pp. [1-4].

“... tomados de Collected poems (London, Albine Press, 1959)".

Traducción de Manuel Moreno Jimeno.

C.: "De la primera fiebre de amor a su plaga".- "Entre aquellos muertos en el bombardeo del amanecer había un hombre de cien años".- "Aquí en esta primavera".- "La mano que firmó el papel".- "Y la muerte no prevalecerá"."Hubo un tiempo". 
14. HELBERG, Heinrich

Libertad.

p. [5].

Poesía.

15. TORO MONTALVO, César

[Poemas.]

Pp. [6-9].

C.: "Salelí y la nueva navidad".- "Matrimonio de la dulce maru y su hermoso okobín cuidador de odres".- "Mimí niña madre de teteras y gorditas".- "Casa de maqui".- "Cuerpo de amapolas".- "Campo de resplandor".

16. BALLÓN, Enrique

Vallejo y los abatanares de la crítica temática.

Pp. [10-14].

Reseña a: James Higgins. Visión del hombre y de la vida en las últimas obras poéticas de César Vallejo. México: Siglo XXI Editores, 1970.

C.: 1. El desorden, supuesto impresionista de axiología.- 2. Comparación no es razón.- 3. La tautología al rango de explicación.- 4. La opacidad como transgresión deb haxiomatđecclafidadLetras

17. SILVA-SANTISTEBAN, Ricardo

\section{"Jorge Puccinelli Converso"}

Perú del corazón.

P. [15].

Comenta la "dedicación del destacado poeta belga Marcel Hennart", como traductor y difusor de la poesía peruana contemporánea.

\section{№ 4. ABRIL DE 1971}

18. PO CHU YI

Balada de la congoja eterna.

Pp. [1-5].

Poesía.

Traducción de Javier Sologuren. 
19. THORNE, Carlos

Apure, Leyva.

Pp. [6-8].

Cuento.

20. BELLI, Carlos Germán

[Poemas.]

Pp. [9-11].

C.: "Cantar primero".- "Cantar II".- "Penas de un bolo alimenticio por su descontentadiza presa de carne en una fría mañana del siglo XVI".

21. BRETON, André

Vida legendaria de Max Ernst. Precedido por una breve discusión sobre la necesidad de un nuevo mito.

Pp. [12-15].

Ensayo

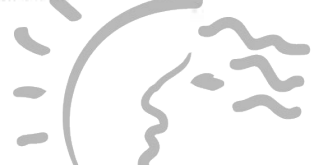

“...tomado de su Le surréalisme et le peinture, 1928-1965. 9. ed., revué et corrigé. Paris, Gallimard, 1965".

Traducción de Javier Sologuren y Armando Rojas "Jorge Puccinelli Converso"

\section{$\mathrm{N}^{\circ}$ 5. MAYO DE 1971}

22. MALLARMÉ, Stéphane

La siesta de un fauno (Versión para la escena).

Pp. [1-7].

Poesía.

"Por primera vez se publican reunidos los fragmentos escénicos de...".

Traducción de Ricardo Silva-Santisteban. 
23. RATTO, Luis Alberto

José María Arguedas, El zorro de arriba y el zorro de abajo.

Pp. [8-9].

Reseña a la novela de José María Arguedas [Buenos Aires: Editorial Losada, 1971].

24. MORENO JIMENO, Manuel

[Conflagraciones del tiempo y de la sangre.]

Pp. [10-12].

"... poemas que pertenecen a la colección... [...]. Los dos últimos poemas en prosa son de un libro aún sin título".

C.: "Después de la sangre".- "La evidencia inacabable".- "El tiempo es oro".- "En el alto nivel".- "Martillada la carne".- "Saeta encarnizada".- "El tesoro del arrapiezo".

25. STRAVINSKY, Igor

A propósito de La consagración de la primavera.

Pp. [13-15].

“... texto que acompaña al álbum Three fayorite ballets de Columbia Masterworks". "Jorge Puccinelli Converso"

Traducción de Ricardo Silva-Santisteban.

\section{No 6. JUNIO DE 1971}

26. SAINT-JOHN PERSE

Para Dante.

Pp. [1-8].

"Discurso del poeta francés en la inauguración del Séptimo Centenario de Dante, en Florencia, el 20 de abril de 1965".

Texto tomado de su Pour Dante, Paris, Gallimard, 1965. 
Traducción de Armado Rojas.

27. UNGARETTI, Giuseppe

Ironia.

P. [8].

Poesía.

Traducción de Javier Sologuren.

28. QUASIMODO, Salvatore

Casi un madrigal.

P. [9].

Poesía.

Traducción de Javier Sologuren.

29. CISNEROS, Antonio

Crónica de viaje / Crónića de viejo.

P. [10-11].

Poesía.

\section{Biblioteca de Letras}

30. BUENO, Raúl (1944)ge Puccinelli Converso"

Obertura.

P. [11].

“... poema que pertenece a la colección Concierto".

31. RAMÍREZ, Luis Hernán

Un mundo dividido de Wáshington Delgado.

Pp. [12-15].

Reseña a: Wáshington Delgado, Un mundo dividido (Poesía 1951-1970).

Lima: Casa de la Cultura del Perú, 1970. 


\section{№ 7. JULIO DE 1971}

32. SOLOGUREN, Javier, Marcel HENNART y Roberto DÁVILA Poesia belga contemporánea.

Pp. [1-7].

“... brevísimo aunque necesario panorama de la poesía belga de lengua francesa en lo que va del siglo. Algunos textos se han traducido de la Anthologie poétique de L'exposition. Bruxelles, 1958".

C.: "Tête silencieuse evoquant les images..." [Cabeza silenciosa que evoca las imágenes..."], Géo Libbrecht [traducción de J.S.].- "La cocina", Maurice Careme [traducción de J.S.].- "Poema de los pequeños acontecimientos", Georges Linze [traducción de M.H. y R.D.].- "Nacimiento del poema", Edmond Vandercamen [traducción de J.S.].- "Veredicto", "En sî", Achille Chavée [traducción de M.H.].- "Breughel", Andrée Sodenkamp [traducción de J.S.].- "La silla", Anne-Marie Kegels [traducción de M.H. y R.D.].- "[Vivo. Indefinible.]", Jacques Izoard [traducción de J.S.].- "Aprisa van los muertos", Franz Hellens [traducción de M.H.] "Me llămo barro", Marcel Hennart [traducción de J.S.].

33. BENDEZÚ, Francisco

[Poemas.] Biblioteca de Letras

Pp. [8-9]. "Jorge Puccinelli Converso"

C.: "Aries" del libro Alquimia.- "Cantábile" de la colección Cicatrices.

34. MARTÍNEZ, Gregorio

La casa de siempre.

Pp. [10-11].

“... primera producción que publica...”.

Cuento.

35. SPENDER, Stephen

[Poemas.]

Pp. [12-13]. 
"Los textos han sido tomados de The edge of Being y Collected poems (1928-1953). London, Faber and Faber Ltd."

Traducción de Ricardo Silva-Santisteban.

C.: "Never being, but always at the edge of Being..." [Sin ser nunca, pero siempre al filo del Ser...]".- "I think continually of fhose who were truly great... [Pienso continuamente en aquellos que de verdad fueron grandes...]"."La vejez de Hoelderlin" .- "Canción de Fausto".- "En Atica".

36. DELGADO, Wáshington

Un mundo para Julius de Alfredo Bryce.

Pp. [14-15].

Reseña a la novela publicada en Barcelona por Barral Editores, en 1970.

37. HOELDERLIN, Friedrich

[Poemas.]

Pp. [1-4].

\section{№ 8. OCTUBRE DE 1971}

C.: "Da ich ein knåbe warte [Cuando yoceracgrsmuchacho...]" (traducción de María Esther Mangariello] "Ganción del destino" [traducción de Heinrich Helberg y Christine Huenefeldt].- "Los dioses" [traducción de Rodolfo E. Modern].- "Die linien des Lebens sind verschieden... [Las líneas de la vida son distintas...]" [traducción de H.H. y Ch.H.].- "Das angenehme dieser Welt hab'ich genossen... [iLo agradable de este mundo lo he gozado...]" [traducción de H.H. y Ch.H.].- "Forma y espíritu" [traducción de H.H. y Ch.H.]."Den nirgen bleibt er... [Porque en ningún lugar se queda...]" [traducción de H.H. y Ch. H.].

38. LOAYZA, Luis

Algún tiempo.

Pp. [5-6].

Cuento. 
39. MICHAUX, Henri

La carta.

P. [7].

“... forma parte de la colección Labyrinthes. Paris: Editions Godet, 1944”.

Traducción de Javier Sologuren.

40. SZYSZLO, Fernando de

[Grabado.]

P. [8].

41. SOLOGUREN, Javier

Estela para Szyszlo.

P. [9]

Poesía.

42. LAUER, Mirko

[El ángel de la anunciación interceptado.]

Pp. [10-13].

c.: "Cromo para iblidateatuable, LeEscenas lacustres de la vieja Barcelona".- "Arte del dialogo interionelliparanuanacátedra (solicitud)".- "Sobre un lugar común francés, italiano, alemán, etc.”.

43. BARTHES, Roland

La fractura del sentido.

Pp. [14-15].

“... texto tomado de L'empire des signes. Genève: Editions d'Arte Albert Skira, 1970”.

Ensayo.

Traducción de Enrique Ballón Aguirre. 
44. DELGADO, Wáshington

[La poesía de Xavier Abril.]

Pp. [1-4].

45. MELIS, Antonio

La poesía de Xavier Abril.

Pp. [5-9].

“...texto tomado de la revista Ad Libitum. Urbino, №. 3, Julio 10 de 1967”.

Traducción de Carlos Germán Belli.

46. ABRIL, Xavier

La rosa escrita [1937].

Pp. [10-13].

Poesía.

C.: "Rosa eterna".- "La rosa de su nombre".- "La rosa 13".- "La rosa humana (Entre sombrßỳ todsa)teca de Letras

47. ABRIL, Xavier

\section{"Jorge Puccinelli Converso"}

Al cisne (Homenaje a Stéphane Mallarmé).

Pp. [13-14].

Poesía.

C.: II.- IV.

48. 'ABRIL, Xavier

A José María Eguren, en su clara noche / en su oscuro cristal [1937]. Pp. [14-15].

Firmado: Costa Azul, Canelones, Uruguay, 1964. 
49. ABRIL, Xavier

Patética olvido (Poema dramático) [1937].

Pp. [16-19].

C.: "Primer cuerpo del poema".- "Segundo cuerpo del poema".- "Tercer cuerpo del poema".- "Cuarto cuerpo del poema".- "Epílogo".

50. ABRIL, Xavier

El autómata.

Pp. [20-24].

Prosa poética.

“... fue escrito entre 1929 y 1930. De él sólo se conocía el capítulo "Lucha y pérdida del mundo", que apareció en la revista Bolívar, Madrid, № 13, Noviembre de 1930".

C.: El silencio.- Origen y presencia del hombre.- Terrible, terrible, terrible.- Metamorfosis de Sergio.

51. ABRIL, Xaviier

Exégesis del poema 'El dominó de José María Eguren. Dedicatoria: A Hugo Friedrich.

Pp. [25-27].

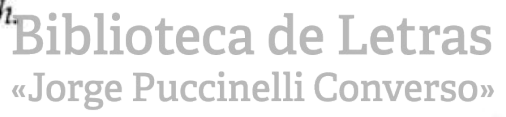

“... comentario que conjuntamente con otros, sobre poemas del mismo Eguren, conforma un libro en preparación”.

52. SILVA-SANTISTEBAN, Ricardo

Contribución a la bibliografía de Xavier Abril.

Pp. [28-31].

C.: Libros.- Poemas no recogidos en libros.- Relatos no recogidos en libros.- Ensayos y prosa diversa no recogidos en Libros.- Sobre Xavier Abril. 
53. DONNE, John

[Poemas.]

Pp. [1-7].

"...tomados de The complete poetry of John Donne, establecida por John T. Shacross. New York, Doubleday, 1967".

Traducción de Ilia Bolaños.

C.: "El éxtasis".- "Los buenos días".- "Canción".- "La salida del sol"."La canonización".- "Despedida prohibiendo el duelo".

54. RIMBAUD, Arthur

Ofelia.

Pp. [8-9].

Poesía.

Traducción de Armando Rojas.

55. REVERDY, Pierre

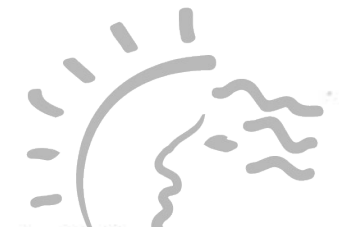

Espacio.

P. [9].

Poesía.

Traducción de Carlos Espinoza.

56. ORTEGA, Julio

Canto de la primera década.

Pp. [10-11].

Poesía.

57. CORNEJO POLAR, Antonio

Sobre La partida inconclusa de Alberto Escobar:

Pp. [12-14]. 
Reseña a La partida inconclusa, o la lectura literaria [Santiago de Chile: Editorial Universitaria, 1970].

58. Crónica de libros.

P. [15].

C.: Robert Graves, La diosa blanca. Traducción, Luis Echávarri. Buenos Aires: 1972. Humphrey Trevelyan, Goethe y los griegos. Traducción, José Paz Garay, rev. Ernesto More. Lima: UNMSM, 1971. Kenzaburo Oe, Un asunto personal. Traducción, Fernando Novoa. Buenos Aires: Editorial Losada, 1971. Emilio Oribe, Rodó, estudio crítico y antología. Buenos Aires: Editorial Losada, 1971. Jayadeva, Gita Govinda. Buenos Aires: Editorial Sudamericana, 1971.

\section{№ 12. FEBRERO DE 1972}

59. DEUSTUA, Raúl Cantos italianos. Pp. [1-4].

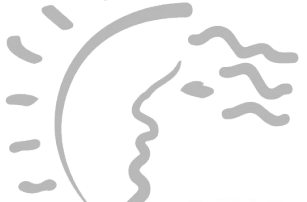

“...poemas escritos en Italia y en el Mediterráneo entre 1956 y 1965".

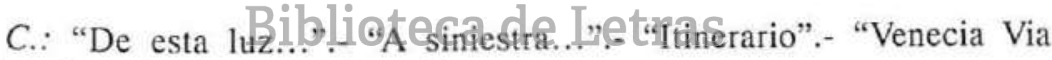
Canaletto".- "Rimbaud Jengtarraftcinelli Converso"

60. R[IVERA] MARTÍNEZ, Edgardo

Elementos.

P. [5].

Poesía.

61. HOELDERLIN, Friedrich

Poemas tardios y de la locura.

Pp. [6-7].

Traducción de Gred Ibscher. 
C.: "En la selva".- "Wast ist Gott?... [¿Qué es Dios]".- "Aber die Sprache... [Mas la palabra...]".- "Was ist der Menschen Le ben?... [¿Qué es la vida de los hombres?.....-- "En tiempos de Sócrates".- "La perspectiva".

62. EIELSON, Jorge Eduardo

Escultura horripilante; homenaje a César Moro y S. Salazar Bondy. Pp. [8-9].

Prosa poética.

"A Javier Sologuren".

63. CISNEROS, Antonio

Dos del British Underground.

Pp. [10-11].

Poetas del movimiento British Underground.

Traducción de Antonio Cisneros.

C.: "Huevo", Spike Hawkins.- "Para S.D.", Lee Harwood.

64. VALÉRY, Paul Biblioteca de Letras
Coloquio en un ser.

Pp. [12-15]. "Jorge Puccinelli Converso"

“...pertenece a la colección Mélange. Ha sido tomado de sus Oeuvres. Paris: Bibliothèque de la Pléiade, 1968, t. I".

Traducción de Manuel Moreno Jimeno.

№ 13. MARZO DE 1972

65. ANÓNIMO

La vigilia de Venus.

Pp. [1-5]. 
"...ha sido tomada de Poetae minores, edición al cuidado de Ernest Raynand. Paris: 1931".

Traducción de Dora Bazán.

66. MARTOS, Marco

[Poemas].

Pp. [6-7].

C.: "Anacoluto".- "Letanía".

Recogidos en su Donde no se ama. Lima: Editorial Milla Batres: 19-20 y 16-17. "Letanía" está recogido con el título "Tu voz".

67. WILLIAMS, Hugo

[Poemas].

P. [8].

Traducción de Mirko Lauer.

C.: "Tras el adiós".- "En un café".- "La ventana abierta".

68. LAUER, Mirko \& Julio ORTEGA

Partitura para una obertura de la lectura de Contra natura.

Pp. [9-15].

Comentario al libro de poesía de Rodolfo Hinostroza [Barcelona: Barral Editores, 1971].

C.: I. 1. Tema. 2. Contexto. 3. Variación. 4. La realité. 5. Envío.- II. 1. ... the lonely boy. 2. De Antonio Cisneros... 3. De Luis Hernández... 4. De Antonio Cisneros... 5. Cazuela del Olimpo. 6. A capella. 7. Coitus.- III: 1. Leitmotiv. 2. Tempo. 3. Fox-trot. 4. Edipo go home. 5. La po-poli-tíca. 6. Coda.- IV. 1. Mentido robador. 2. Ma questo... 3. Summa. 4. Caza del gorrión.- V. 1. Mais passons. 2. Recitativo. 3. Babel. 4. Segundo recitativo.- VI. 1. Xauxa. 2. Reclamo. 3. Un sens plus pur. 4. Política. 5. Historia. 6. Ve, canción. 
69. NERVAL, Gérard de

Ensueño de Carlos VI.

P. [15].

Poesía.

Traducción de Ricardo Silva-Santisteban.

\section{$\mathrm{N}^{2}$ 14. JUNIO DE 1973}

70. ROJAS, Armando

Montes.

Pp. [1-7].

Poesía.

71. SOLOGUREN, Javier

Poesía china.

Pp. [8-10].

Traducción de Javier Sologuren.

C.: "Un canto en la ribera", Li Tai Pa (701-762).- "La ascensión", Tu Fu (712-770).- "El pabellon de la grulla amarilla", Ts" uei Hai (754).- "Regalo de un espejo a manera de adiós”, Po Chu Yi (772-846).

72. OLIVEROS, Alejandro

[Poemas.]

P. [11].

C.: "Helechos".- "Plaza II".

73. GOYEN, William

Tres artificios.

Pp. [12-13].

“...tomado de Poetry. Vol. 74. May. 1949". 
Prosa poética.

Traducción de Ricardo Silva-Santisteban.

C.: "I. Saluda al mar".- "II. descubre las telarañas".- "III. De mañana camina por la playa".

74. COBO BORDA, J.G.

[Consejos para sobrevivir.]

Pp. [14-15].

C.: "Sobre épica II".- "Fechas que siempre vuelven".- "Poética".

75. ARP, Jean

Violetas rojas.

P. [16].

Poesía.

"...escrito en 1945 y apareció en Sede del aire".

Traducción de Javier Sologuren.

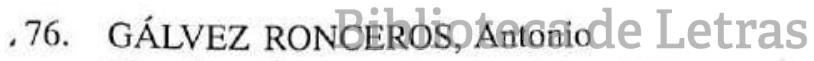

Tre clase de souyoutgosPouentoseniegros.pverso"

Pp. [17-19].

C.: Introducción.- "Burra negra".- "La cólera".- "Tre clase de so"."Jutito".

\section{№ 15 AGOSTO DE 1973}

77. YEATS, William Butler

La torre.

Pp. [1-6].

Firmado: 1926. 
“... tomado de Collected poems de William Butler Yeats. London: Mac Millan \& Co. Ltd., 1965".

Traducción de Ricardo Silva-Santisteban.

C.: "I".- "II".- "III".- Nota del autor.

78. RAZZETO, Mario

[Crónica del extranjero].

Pp. [7-8].

“...poemas que pertenecen a...”.

C.: "Otoño".- "Hablo de los lugares comunes".- "Puente de los Suspiros".- "La vida.

79. JARRELL, Randall

La muerte del artillero del lorreón.

P. [9].

Poesía.

Traducción de Ilia Bolaños.

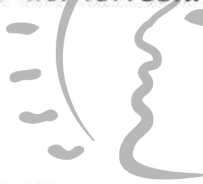

\section{Biblioteca de Letras}

"Poeta norteamericano:"Puccinelli Converso"

80. GILL, Claes

[Poemas.]

Pp. [10-11].

“...tomados de Fragment av et magisk liv [Fragmentos de una vida mágica] (1972) y Ord I jaern [Palabras de hierro] (1942)".

Traducción directa del noruego por Helen Orvig de Salazar. "María".

C.: "El poeta elogia el amor de Deirdre y Naisi y habla de su temor".- 
81. ARISTEGUIETA, Jean

[Poemas.]

Pp. [12-13].

C.: "Cuerpo de la noche".- "Latitud".- "Pasa el silencio".

82. BUDIGNA, Luciano

Aniversario.

P. [14].

"El poema $[\ldots]$ del poeta italiano ha sido tomado de Da sera a notte...".

Traducción de Javier Sologuren.

83. GAZZOLO, Ana María

Estructura de un poema de Carlos Germán Belli: "Sextina del mea culpa".

Pp. [15-19].

$\mathrm{N}^{2}$ 16. SEPTIEMBRE DE 1973

84. AIKEN, Conradiblioteca de Letras [Poemas.]

Pp. [1-7].

"Jorge Puccinelli Converso»

"La selección ha sido tomada de la $2^{\mathrm{a}}$. ed. de Collected poems. New York: Oxford University, 1970".

Traducción de Mercedes Ibáñez Rosazza.

C.: "Preludio XIV".- "Preludio XIX".- "Preludio para Memnon o preludios para la actitud".- "Preludios para la definición tiempo en la roca XCIII"." 'South end".

85. OVIEDO, José Miguel

Falsos haikús del verano.

Pp. [8-10]. 
“...poemas de Izumi Shikibu Nikki [Diario de Izumi Shikibu], famoso diario poético japonés que data aproximadamente del año 1005 de nuestra era...".

Traducción de José Miguel Oviedo.

C.: "Falsos haikús del verano", J.M.O.- "1. La fina gaviota...".- "2. Soledad total...".- "3. Por la mañana...".- "4. Sucesión de olas...".- "5. Expreso mis deseos...".- "6. Nada es comparable...".- "7. La fealdad del mundo..."."8. Pocas cosas...".- "9. El ruido del mar...".-"10. Me duermo...".-"11. Visitantes en la casa...".- "12. Mi mujer decide...".- "13. La rutina se define...".- "14. Blanca y delgada...".- "15. Un deseo nítido...".- "16. La gente va...”.- “17. Llega una nueva pareja...".-"18. Por pura impaciencia...”.-"19. Desperdicié mi juventud...".

86. PEÑA BARRENECHEA, Ricardo

Instancias de la angustia.

Pp. [11-14].

“... es uno de los libros del poeta peruano [...] que había permanecido injustamente inédito. Fue escrito en 1931".

\section{c.: "Canto I".- "GahtöH":eca de Letras}

87. HAGERUP, Inger "Jorge Puccinelli Converso"

[Espera. $]$

P. [15].

"El poema ha sido tomado de Videre. Stockholm, A. Norsted \& Soner Forlag, 1944."

Traducción de Javier Sologuren.

88. FREIRE, Luis

[Relatos.]

Pp. [16-19].

C.: "1".- "2".- "3".- "4".- "5". 
89. PHILL, Abraham

Poemas.

Pp. [20-23].

Traducción de Abelardo Oquendo.

C.: "Introducción", H.G. Reynolds.- "Del tiempo.- "Defensa".- "Paisajes".- "Temperatura".- "Humor".- "El viajero".- "Cena".

90. VARELA, Blanca

Camino a Babel.

Pp. [1-5].

Poesía.

91. SITWELL, Edith

La locura de Saúl.

Pp. [6-9].

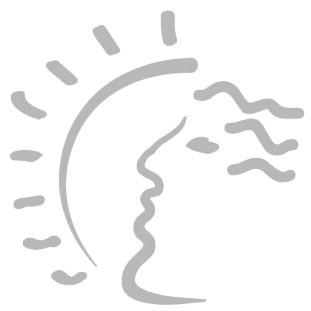

“...poema dramâticicổinconclusa de la poetisa inglesa...”.

Traducción de Manuel Moreno Jimeno.

92. FERRARI, Américo

Sonetos para terminar:

Pp. [10-11].

C.: "[yo ya no...]".-- "[agitando soneto...]".- "[de amorfo y nada...]"."[hacedor de distancia...]".

93. SILVA-SANTISTEBAN, Ricardo

Terra incognita.

Pp. [12-13].

C.: "Romance".- "Retorno". 
94. QUESSEP, Giovanni

[Poemas en prosa.]

P. [14].

“...joven poeta colombiano...".

C.: "Blanco para morir".- "Poderes de la magia o de la luna".

95. HEIDEGGER, Martín

Signos.

Pp. [15-16].

Firmado: Friburgo de Brisgovia, setiembre de 1969.

Ensayo filosófico.

“...publicado en el periódico Neue Zürcher Zeitung en 1969”.

Traducción directa del alemán por David Sobrevilla.

96. VALENTE, José Angel

Tamiris el Tracio.

P. [16].

Poesía.

\section{Biblioteca de Letras} "Jorge Puccinelli Converso»

“...poeta español...”.

97. AUDEN, Wynstan Hugh

[Poemas.]

Pp. [17-23].

C.: "Musée des Beaux Arts" [traducción, Fernando de Szyszlo].- "En memoria de W.B. Yeats" [traducción, Fernando de Szyszlo].- "En honor de la piedra caliza" [traducción, Mercedes Ibáñez Rosazza].- "No, Platón, por favor" [traducción, José Miguel Oviedo].- "Carta póstuma a Gilbert White" [traducción, José Miguel Oviedo]. 
"Los tres primeros poemas han sido tomados de The collected shortes poems (1927-1957) y los dos restantes de números de The New York Review of Books de 1973".

\section{№ 18 AGOSTO DE 1974}

98. HOPKINS, Gerard Manley

El naufragio del Deutschland.

Pp. [1-10].

"...tomado de la 3". ed. de Poems of Gerard Manley Hopkins. Oxford, 1964".

Traducción de Maruja Silva Villacorta.

C.: Primera parte.- Segunda parte.

99. YURKIEVICH, Saúl

La serenisima.

P. [11].

Poesía.

100. OJEDA, Juan "Jorge Puccinelli Converso"

\section{Biblioteca de Letras}

[Diario de navegantes.]

Pp. [12-15].

Poesía.

C.: "Van Gogh en Arles" .- "Meditación de Meister Eckhart"."Mutanabbi".

101. PETÖFI, Sandor

[Poemas.]

Pp. [16-21].

Traducción de Wáshington Delgado. 
C.: "El.- "De nuevo escucho el canto de la alondra".- "La llanura" .- "La estepa en invierno".

102. CALDERÓN FAJARDO, Carlos

El peregrino.

P. [22].

Relato.

103. RODRÍGUEZ REA, Miguel Angel

Arbol.

P. [23].

Poesía.

\section{№ 19. JUNIO DE 1976}

104. RIVERA MARTINEZ, J. Edgardo

Amaru.

Pp. [1-5].

Relato.

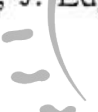

\section{Biblioteca de Letras}

105. SILVA-SANTISTEBANDRicaindelli Converso»

Poesía italiana.

Pp. [6-12].

"A Roberto Paoli".

Traducción de Ricardo Silva-Santisteban.

C.: Guido Cavalcanti, "Madrigal".- Dante Alighieri, "Tanto gentile e tanto onesta pare... [Se muestra tan gentil y recatada]".- Francesco Petrarca, "Gli occhi di ch'io parlai si caldamente... [Los ojos de que hablé cálidamente...]".- Matteo Maria Boiardo, "Gia vidi uscir di l'onde una mattina... [De entre las ondas vi en la hora primera...]".- Ludovico Ariosto, "La verginella e simile alla' rosa... [Semejante es la virgen a la rosa...]".- Torcuato Tasso, "Tacciono i boschi e fiumi... [Los bosques y los ríos enmudecen]".- Giacomo Leopardi, "El infinito".- Dino Campana, "Genovesa".- Giuseppe Ungaretti, 
"La isla".- Eugenio Montale, "Meriggiare pallido e assorto... [Pálido y absorto, sestear...]".- Salvatore Quasimodo, "Y es noche de pronto", "Elegía".Mario Luzi, "Epistolium".

106. ROSE, Juan Gonzalo

Romance de cara al cielo.

P. [13].

Poesía.

107. CÉSAR MORO

Cartas de amor.

Pp. [14-18].

“...escritas en México...”.

Prosa poética.

Se incluyen cinco cartas.

108. LASTRA, Pedro

Del sentimiento de equidistancia. (Para una poética del lector).

P. [19].

Ensayo.

109. SAFO

[Poemas.]

Pp. [20-21].

Traducción de Ana María Gazzolo.

C.: "A Afrodita".- "Al amado".

110. PETRARCA, Francesco

Canción.

Pp. [22-23].

Traducción de Javier Sologuren. 
111. MONTES DE OCA, Marco Antonio

[Poemas].

P. [24].

C.: "Así es la cosa".- "Recuento".

112. CERVANTES, Francisco

[Poemas.]

P. [25].

C.: "Otoño sin perdón".- "Retoño del triclinio invernal".

113. BERRYMAN, John

[Poemas.]

Pp. [26-27].

Traducción de Antonio Cisneros.

C.: "Paisaje de invierno".- "Nota a Wang Wei".

114. GAZZOLO, Ana María

[Poemas.]

Pp. [28-29]. Biblioteca de Letras

C.: "Esta hora...".-. "Sốlo quería llorar sola..."."." "Una hora....- "Blanco el rostro...".- "La razón de la nada...".- "Insistente frío...".- "Llegar a la espina...".- "Piedra fría...".

115. ORELLANA, Carlos

Pobre Paca.

Pp. [30-33].

"...pertenece al libro La mar que es el morir".

Cuento.

116. ZISMAN, Alex

El sol de Lima de Luis Loayza.

Pp. [34-37]. 
Reseña a este libro de ensayos [Lima: Mosca Azul Editores, 1974].

\author{
№. 20. AGOSTO DE 1977 \\ HOMENAJE A EMILIO ADOLFO WESTPHALEN
}

117. VARGAS LLOSA, Mario

Presentación.

Pp. [1]-2.

118. PEÑA BARRENECHEA, Enrique

En el homenaje a Emilio Adolfo Westphalen.

Pp. 3-4.

119. MORENO JIMENO, Manuel

Presencia de Emilio Adolfo Westphalen.

P. 5.

120. OVIEDO, José Miguel-

Palabras para Westphalen.

Pp. 6-8.

121. BAcIÚ, Stefan Biblioteca de Letras

Emilio Adolfo Westphatenu poèncele (co tortuga voladora.

Pp. 9-13.

122. BELLI, Carlos Germán

Westphalen, el abstencionista.

Pp. 14-15.

123. ORTEGA, Julio

Una nota sobre Westphalen.

Pp. 16-17.

124. CUETO, Alonso

El primer surrealista.

Pp. 18-20. 
125. CÉSAR MORO

Westphalen.

P. 21.

Poesía.

126. EIELSON, Jorge Eduardo

Westphalen dice.

Pp. 21-22.

Poesía.

127. CISNEROS, Antonio

Después de corregir las pruebas de Amaru en la imprenta-1967 [Emilio Adolfo Westphalen].

P. 22.

Poesía.

128. ROJAS, Armando

Lectura de Westphalen.

Pp. 23-26.

Poesía.

\section{Biblioteca de Letras} "Jorge Puccinelli Converso"

129. WESTPHALEN, Emilio Adolfo

[Poemas.]

Pp. 27-29.

C.: "Nerval y el amor".- "Libre".- "Poema inútil".

130. SOLOGUREN, Javier

Los últimos poemas de E.A. Westphalen.

Pp. 30-31.

131. SILVA-SANTISTEBAN, Ricardo

Westphalen, poeta de los elementos.

Pp. 32-37. 


\section{GONZÁLEZ VIGIL, Ricardo}

Westphalen o el esplendor de la imaginación lírica.

Pp. 38-43.

"Comentario al quinto poema de Las ínsulas extrañas".

133. FERRARI, Américo

Lectura de Abolición de la muerte.

Pp. 44-48.

134. VERÁSTEGUI, Enrique

Una flor en revuelta, cf. Abolición de la muerte.

Pp. 49-59.

135. RIVERA MARTÍNEZ, Edgardo

El pensamiento de Westphalen.

Pp. 60-69.

136. PODESTÁ, Bruno

Las revistas de Emilio Adolfo Westphalen.

Pp. 70-72.

\section{SILVA-SANTISTEBAN, Ricardo}

Bibliografia de Emilio Adolfo Westphalen.

Pp. 73-79.

C.: Libros.- Poemas no recogidos en libros.- Versiones poéticas.- Artículos, ensayos y prosa diversa no recogidos en libros.- Sobre Emilio Adolfo Westphalen. En libros. En publicaciones periódicas. 


\section{ÍNDICE ONOMÁSTICO}

Abril, Xavier 44, 45, 46-51

Aiken, Conrad 84

Alighieri, Dante 26, 105

Apollinaire, Guillaume 3

Arguedas, José María 23

Ariosto, Ludovico 105

Aristeguieta, Jean $\mathbf{8 1}$

Arp, Jean $\mathbf{7 5}$

Baciú, Stefan 121

Ballón Aguirre, Enrique 16, 43

Barthes, Roland 43

Bazán, Dora 65

Belli, Carlos Germán 5, 20, 45, 83, 122

Bendezú, Francisco 33

Berryman, John 113

Boiardo, Matteo Maria 105

Bolaños, Ilia 53, 79

Breton, André 21

Breughel, Peter, el Viejo 32

Bryce Echenique, Alfredo 36

Budigna, Luciano 82

Bueno, Raúl 30
Auden, Wynstan Hugh 97

Delgado, Wáshington 31, 36, 44, 101

Deustua, Raúl 59

Dop, David 11

Donne, John 53

Echávarri, Luis 58

Eguren, José María 4, 48, 51

Eielson, Jorge Eduardo 1, 62, 126

Ernst, Max 21

Escobar, Alberto 57

Espinoza, Carlos 55

Ferrari, Américo 92, 133

Freire, Luis 88

Friedrich, Hugo 51

Galvez Ronceros, Antonio 76

Garcilaso de la Vega, el Inca 10

Gazzolo, Ana María 83, 109, 114

Gill, Claes 80

González Vigil, Ricardo 132

Goyan, William 73

Graves,-Robert 58

"Jorge PuccinelliguevaraılBablo 9

Hagerup, Inger 87

Calderón Fajardo, Carlos 102

Campana, Dino 105

Careme, Maurice 32

Carlos VI 69

Cavalcanti, Guido 105

Cervantes, Francisco 112

César Moro 62, 107, 125

Chavée, Achille 32

Cisneros, Antonio 29, 63, 68, 127

Cobo Borda, Juan Gustavo $\mathbf{7 4}$

Cornejo Polar, Antonio 57

Cueto, Alonso 124

Damas, León D. 11

Dávila, Roberto 32
Harwood, Lee 63

Hawkins, Spike 63

Heidegger, Martin 95

Helberg, Heinrich 14, 37

Hellens, Franz 32

Hennart, Marcel 17, 32

Hernández, Luis 68

Higgins, James 16

Hoelderlin, Friedrich 35, 37, 61

Hopkins, Gerard Manley 98

Huenefeldt, Christine 37
Hinostroza, Rodolfo 68 
Ibáñez Rosazza, Mercedes 84, 97

Ibscher, Gred 61

Izoard, Jacques 32

Jarrell, Randall 79

Jayadeva 58

Joyce, James 6

Kegels, Anne-Marie 32

Lastra, Pedro 108

Lauer, Mirko 12, 42, 67, 68

Leopardi, Giacomo 105

Libbrecht, Géo 32

Linze, Georges 32

Li Tai Po 71

Loayza, Luis 10, 38, 116

Luzi, Mario 105

Mallarmé, Stéphane 22, 47

Mangariello, María Esther 37

Martinez, Gregorio 34

Martos, Marco 66

Melis, Antonio 45

Michaux, Henri 39

Modern, Rodolfo

Montale, Eugenio 105 «Jorge PuccinelliRodó, José Ennique 58

Montes de Oca, Marco Antonio 111

More, Ernesto 58

Moreno Jimeno, Manuel 13, 24, 64, 91, 119

Motokiyo, Seami 2

Nerval, Gérard de 69

Novoa, Fernando 58

Oe, Kenzaburo 58

Ojeda, Juan 100

Oliveros, Alejandro 72

Oquendo, Abelardo 12, 89

Orellana, Carlos 115

Oribe, Emilio 58

Ortega, Julio 56, 68, 123
Orvig de Salazar, Helen $\mathbf{8 0}$

Oviedo, José Miguel 11, 85, 97, 120

Palma, Ricardo 10

Paoli, Roberto 105

Paz Garay, José 58

Peña Barrenechea, Ricardo 86, 118

Perse, Saint-John 26

Petöfi, Sandor 101

Petrarca, Francesco 105, 110

Phill, Abraham 89

Po Chu Yi 18, 71

Podestá, Bruno 136

Quasimodo, Salvatore 28, 105

Quessep, Giovanni 94

Ramírez, Luis Hernán 31

Ratto, Luis Alberto 23

Raynand, Ernest 65

Razzeto, Mario 78

Redondeau 2

Reverdy, Pierre 55

Rimbaud, Arthur 54

Rivera Martínez, J. Edgardo 66, 104,

de 135 ras

Rodríguez Rea, Miguel Angel 103

Rojas, Armando 5, 12, 21, 54, 70, 128

Rose, Juan Gonzalo 106

S.D. 63

Safo 109

Salazar Bondy, Sebastián 62

Seferis, Georges 8

Senghor, Leopold Sedar 11

Shacross, John T. 53

Silva-Santisteban, Ricardo 3, 6, 7, 17, $22,3552,69,73,77,93,105,131$, 137

Silva Villacorta 98

Sitwell, Edith 91

Sobrevilla, David 95 
Sodenkamp, Andrée 32

Sologuren, Javier 2, 8, 18, 21, 27, 28, $32,39,41,71,75,87,110,130$ Spender, Etephen 35

Stravinsky, Igor 25

Szyszlo, Fernando de 40, 41, 97

Tasso, Torcuato 105

Thomas, Dylan 13

Thorne, Carlos 19

Toro Montalvo, César 15

Trevelyan, Humphrey 58

Ts'suei Hai 71

Tu Fu 71

Ungaretti, Giuseppe 27, 105
Valente, José Angel 96

Valéry, Paul 64

Vandercamen, Edmond 32

Varela, Blanca 90

Vargas Llosa, Mario 117

Verástegui, Enrique 134

Westphalen, Emilio Adolfo 118-123, $125-128,129,130,131,135-137$

Williams, Hugo 67

Yeats, William Butler 77

Yurkievich, Saúl 99

Zisman, Alex 116

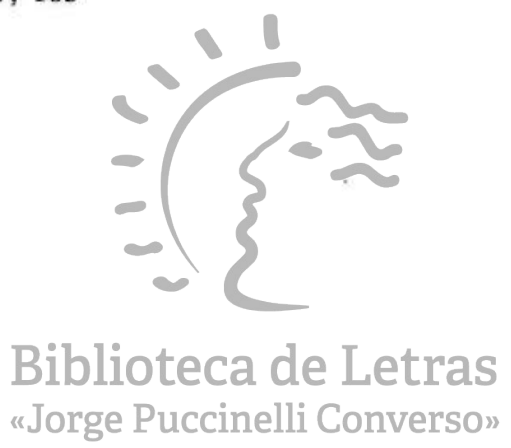

